Pathologe 2022 $43: 45-50$

https://doi.org/10.1007/s00292-021-01016-6

Angenommen: 24. September 2021

Online publiziert: 1. November 2021

○ Der/die Autor(en) 2021, korrigierte Publikation 2021

Schwerpunktherausgeber

H.I. Grabsch, Maastricht, Niederlande

R. Langer, Linz, Österreich

M. Vieth, Bayreuth

\section{Tumor Budding beim kolorektalen Karzinom - Informationen zur klinischen Anwendung und Anleitung zur praktischen Bestimmung}

\author{
Felix Müller · Alessandro Lugli · Heather Dawson \\ Institut für Pathologie, Universität Bern, Bern, Schweiz
}

Zusammenfassung

\section{In diesem Beitrag}

- Hinführung

- Anwendungsbereich pT1 CRC

- Anwendungsbereich Stadium-II-CRC

- Anwendungsbereich in präoperativen Biopsien

- Ungeklärte und/oder schwierige Situationen bei der Tumor-Budding-Bestimmung

- Ausblick nach der ITBCC 2016

Hintergrund: Bei einzelnen Patienten mit kolorektalen Karzinomen (CRC) zeigt sich ein schlechter klinischer Verlauf innerhalb desselben UICC-Stadiums (Union for International Cancer Control). Die Identifizierung von zusätzlichen Risikofaktoren ist daher notwendig, um eine optimale Therapieplanung zu erreichen.

Fragestellung: In welchen Situationen kann Tumor Budding die klinische Therapieentscheidung beeinflussen und wie sollte die standardisierte Auswertung erfolgen?

Material und Methode: Aktuelle Publikationen zum Thema Tumor Budding werden mit Fokus auf die praktische Anwendung und potenzielle Problemfälle in der Bestimmung des Tumor Buddings erläutert.

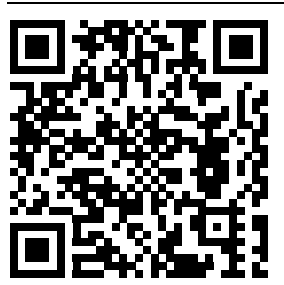

QR-Code scannen \&Beitrag online lesen

Ergebnisse: Tumor Budding ist ein signifikanter Risikofaktor für einen schlechteren Verlauf des CRC und kann bei pT1-Karzinomen sowie Stadium-II-Karzinomen die Behandlung beeinflussen. Die Auswertung wurde durch die International Tumor Budding Consensus Conference (ITBCC) 2016 standardisiert und ist in der Praxis anwendbar. Schwierigkeiten in der Anwendung können durch die Kenntnis von potenziellen Problemfällen vermieden werden.

\title{
Schlüsselwörter
}

Lymphknotenmetastasierung · Epithelial-mesenchymale Transition · Tumorzellenverbände · Therapieplanung · Tumorgraduierung

\section{Hinführung}

Das kolorektale Karzinom (CRC) ist eine weltweit auftretende maligne neoplastische Erkrankung, welche mit einer hohen Mortalität assoziiert ist [9]. Der Standard zur Klassifizierung stellt die TNMKlassifikation („tumour, node, metastasis“) der Union for International Cancer Control (UICC) dar. Innerhalb gleicher pT-Stadien zeigen sich unterschiedliche klinische Verläufe bei einzelnen Risikopatienten, welche beispielsweise bereits früh Lymphknotenmetastasen entwickeln [1]. Die Identifizierung von Hochrisikogruppen, welche von einer ausgedehnteren Therapie profitieren, ist daher von großer Bedeutung.
Tumor Budding ist ein etablierter unabhängiger Risikofaktor für einen aggressiveren Verlauf und kann in bestimmten klinischen Szenarien ein Hilfsmittel sein, um eine Entscheidung für weitere Therapien in Form von chirurgischer Resektion oder adjuvanter Chemotherapie zu treffen [17].

\section{Einleitung}

Tumor Budding beschreibt den Effekt von diskohäsivem Wachstum einzelner Tumorzellen bis zu Verbänden $<5$ Tumorzellen im tumorassoziierten Stroma der Infiltrationszone verschiedener Karzinome (s. Infobox für die Definition eines Tumor Buds [17]). Der Effekt wurde erstmal von 
Tab. 1 Ablaufschema für die Bestimmung des Tumor Budding Scores [14]

\begin{tabular}{|l|l|}
\hline 1 & $\begin{array}{l}\text { Ein Hämatoxylin/Eosin- (HE-)Schnitt mit dem höchsten Grad an Tumor Budding peritu- } \\
\text { moral wird für die Bestimmung ausgewählt (Panzytokeratinimmunhistochemie kann } \\
\text { helfen, entsprechende Hotspot-Areale zu identifizieren) }\end{array}$ \\
\hline 2 & $\begin{array}{l}\text { Zehn Felder mit 10-facher Vergrößerung werden hinsichtlich des lokalen „Hotspots" } \\
\text { untersucht }\end{array}$ \\
\hline \multirow{2}{*}{3} & $\begin{array}{l}\text { Das Hotspot-Areal wird mittels 20-facher Vergrößerung betrachtet und die Tumor Buds } \\
\text { ausgezählt }\end{array}$ \\
\hline \multirow{2}{*}{4} & $\begin{array}{l}\text { Das Ergebnis wird durch den Normalisierungsfaktor (- Tab. 2) geteilt, um eine Korrektur } \\
\text { für verschiedene Öffnungsgrößen des Okulars des Mikroskop zu erreichen }\end{array}$ \\
\hline \multirow{2}{*}{5} & $\begin{array}{l}\text { Das normalisierte Ergebnis ermöglicht die Einordnung in die drei Tumor-Budding-Kate- } \\
\text { gorien: }\end{array}$ \\
\cline { 2 - 3 } & Bd1 (0-4) „low“ \\
\cline { 2 - 2 } & Bd2 (5-9) „intermediate“ \\
\cline { 2 - 2 } & Bd3 (10 oder mehr) „high“ \\
\hline
\end{tabular}

\begin{tabular}{|l|l|l|}
\hline \multicolumn{3}{|l|}{ Tab. 2 Normalisierungsfaktoren [3] } \\
\hline $\begin{array}{l}\text { Okular Augenöffnungsdurch- } \\
\text { messer }(\mathbf{m m})\end{array}$ & $\begin{array}{l}\text { Betrachtete Fläche } \\
\left(\mathbf{m m}^{2}\right)\end{array}$ & $\begin{array}{l}\text { Normalisierungsfaktor zu } \\
\mathbf{0 , 7 8 5} \mathbf{~ m}^{\mathbf{2}}\end{array}$ \\
\hline 18 & 0,636 & 0,81 \\
\hline 19 & 0,709 & 0,903 \\
\hline 20 & 0,785 & 1 \\
\hline 21 & 0,866 & 1,103 \\
\hline 22 & 0,95 & 1,21 \\
\hline 23 & 1,039 & 1,323 \\
\hline 24 & 1,131 & 1,440 \\
\hline 25 & 1,227 & 1,563 \\
\hline 26 & 1,327 & 1,69 \\
\hline $\begin{array}{l}\text { Anzahl Tumor Buds (bei 20-facher Vergrößerung)/Normalisierungsfaktor (gemäß Durchmesser Oku- } \\
\text { lar)= Tumor Buds (normalisiert) }\end{array}$ & \multicolumn{2}{|l}{} \\
\hline
\end{tabular}

Imai et al. [17] in den 1950er-Jahren als „Aussprossung" bezeichnet [15]. Tumor Budding kann im Übergangsbereich von Normalgewebe zum Karzinom als Zeichen der Infiltration (peritumorales Budding) und innerhalb eines Karzinoms (intratumorales Budding) beobachtet werden.

Im CRC konnte Tumor Budding als unabhängiger prognostischer Faktor durch zahlreiche Studien bestätigt werden [17, 22]. Es zeigte sich ebenfalls eine Korrelation mit höherem Tumorgrad, lymphovaskulärer Infiltration, Lymphknotenmetastasen und Fernmetastasen.

Als Ursache für diesen migratorischen Effekt von Tumorzellen wird die epithelialmesenchymale Transition (EMT) postuliert, bei welcher epitheliale Zellen eine geringere Zell-Zell-Adhäsion durch Verlust von E-Cadherin zeigen und vermehrt Marker von mesenchymalen Zellen wie Vimentin, SMA ("smooth muscle actin“) oder Fibronektin exprimieren [13]. Es handelt sich hierbei um einen mehrstufigen Pro- zess, bei welchem die Zellen von epithelial zu mesenchymal transformieren und welcher durch die Aktivierung der Wnt/ $\beta$-Catenin-Pathways bei Verlust von membranärer E-Cadherin-Expression gekennzeichnet ist. In der aktuellen Forschung wird angenommen, dass Tumor Buds eine partielle epithelial-mesenchymale Transition (pEMT) darstellen, da etwa bei Tumor Buds des duktalen Adenokarzinoms des Pankreas keine Korrelation zwischen der membranären Reduktion von E-Cadherin und der Expression von Vimentin besteht [13].

Die EMT kann außerhalb der Karzinogenese auch bei physiologischen Prozessen, wiez.B. derEmbryogenese oder der Wundheilung, beobachtet werden. Die aberrante Expression von EMT-assoziierten Markern bei Karzinomen wird als Kennzeichen für ein erhöhtes Metastasierungsrisiko betrachtet [18].

Auch wenn Tumor Budding schon länger insbesondere beim CRC im Fokus zahlreicher Studien stand, war die Anwendung in der Praxis bis vor wenigen Jahren wegen der nicht standardisierten Auswertung limitiert. Im Rahmen der „International Tumor Budding Consensus Conference" (ITBCC) wurde ein 2016 ein Konsens zur Auswertung des Tumor Budding mit einheitlichen Empfehlungen definiert, welche die Standardisierung der Auswertung und somit die Aufnahme in die Leitlinien ermöglichte (zusammengefasst in - Tab. 1 und $2 ;[14,17])$. Seither wurde Tumor Budding als zusätzlicher Risikofaktor für die Progression des CRC in die Guidelines der UICC [4], den „College of American Pathologists"- (CAP-)Richtlinien [10], den "National Comprehensive Cancer Network"(NCCN-)Richtlinien [2], der deutschen S3Leitlinie [3] und bei den „European Society for Medical Oncology consensus"- (ESMO)Richtlinien [5] aufgenommen.

Bei der ITBCC 2016 wurden folgende Aussagen definiert [17]:

1. Tumor Buds sind einzelne Tumorzellen oder Zellverbände $<5$ Zellen.

2. Tumor Budding ist ein unabhängiger Prädiktor für das Auftreten von Lymphkotenmetastasen bei pT1Karzinomen.

3. Tumor Budding ist ein unabhängiger Prädiktor für das Überleben bei Stadium-II-Karzinomen.

4. Tumor Budding sollte neben anderen klinischen und pathologischen Faktoren in einem multidisziplinären Team diskutiert werden.

5. Tumor Budding wird an Hämatoxylin/ Eosin- (HE-)Schnitten bestimmt.

6. Intratumorales Tumor Budding tritt bei CRC auf und erhöht das Risiko für Lymphknotenmetastasen.

7. Tumor Budding wird in einem einzelnen "Hotspot" $\left(0,785 \mathrm{~mm}^{2}\right)$ in der Infiltrationszone bestimmt.

8. Drei Kategorien sollten verwendet werden (Bd1 [0-4] „low“; Bd2 [5-9] „intermediate“; Bd3 [ $\geq 10]$ „,high")

9. Tumorgraduierung und Tumor Budding sind unterschiedliche morphologische Charakteristika.

10. Tumor Budding sollte in die Leitlinien/ Protokolle für Tumorberichte bei CRC integriert werden.

In einer retrospektiven Studie an $771 \mathrm{~Pa}$ tienten mit CRC konnte gezeigt werden, dass Gradierung und Tumor Budding zwei 


\section{Infobox 1}

Definition Tumor Bud [17]

Tumor Buds sind einzelne Tumorzellen oder kleine Tumorzellverbände $<5$ Zellen, welche sich im Tumorzentrum (intratumorale Tumor Buds, ITB) oder peritumoral (peritumorale Tumor Buds, PTB) nachweisen lassen. Sie sind abzugrenzen von "poorly defined clusters": Tumorzellenverbänden von $\geq 5$ Zellen, welche kein glanduläres Wachstumsmuster zeigen und ebenso intra- oder peritumoral auftreten können.

statistisch unabhängige Größen sind, welche beide ein höheres pT-Stadium, Lymphangioinvasion und Fernmetastasen vorhersagen, jedoch Tumor Budding ein besserer prädiktiver Wert für das krankheitsfreie und Gesamtüberleben der Patienten ist. Gradierung und Tumor Budding sind daher nicht gleichzusetzen und können unterschiedliche biologische Prozesse darstellen [28].

Tumor Budding konnte neben dem CRC auch in verschiedenen anderen Karzinomen als relevanter Risikofaktor für einen aggressiveren Verlauf identifiziert werden, so etwa u. a. beim Plattenepithelkarzinom des Ösophagus, dem duktalen Adenokarzinom des Pankreas, dem intestinalen Typ des Magenkarzinoms, dem endometroiden Adenokarzinom des Uterus oder verschiedenen Plattenepithelkarzinomen des Kopf/Hals-Bereichs [21, 27].

\section{Anwendungsbereich pT1 CRC}

Kolorektale Karzinome im pT1-Stadium können mittels endoskopischer Entfernung mit oder ohne onkologische Resektion (mit Lymphadenektomie) behandelt werden [3].

Grundsätzlich wird eine chirurgische Therapie bei erhöhtem Risiko für Lymphknotenmetastasen favorisiert. Das Risiko für Lymphknotenmetastasen beim pT1CRC variiert zwischen $<1$ und ca. $35 \%$ und ist abhängig von histologisch fassbaren Risikofaktoren wie Gradierung, Gefäßinvasion, Tumorinfiltrationstiefe und Tumor Budding, wobei das Risiko mit multiplen Risikofaktoren steigt [25].

In Metaanalysen konnte gezeigt werden, dass die Odds Ratio von Tumor Budding für Lymphknotenmetastasen bei pT1Karzinomen zwischen 6,44 und 7,74 liegt $[7,8,11]$ und somit zumindest ebenso stark zum Risiko für Lymphknotenmetastasen beiträgt wie eine Lymphangioinvasion, Infiltrationstiefe und Gradierung.

In den Empfehlungen der deutschen S3-Leitlinien wird eine chirurgische Therapie bei "high risk" pT1-Karzinomen empfohlen, welche entweder Lymphangioinvasion, hohes Grading, Tumor Budding $(\mathrm{Bd} 2$ oder $\mathrm{Bd} 3)$ aufweisen [3].

\section{Anwendungsbereich Stadium-II- CRC}

Bei Patienten mit lokal fortgeschrittenem CRC im Stadium Il gemäß UICC konnte in einem systematischen Review mit insgesamt 1652 inkludierten Patienten nachgewiesen werden, dass die Gesamtüberlebenszeit bei gleichzeitigem Auftreten von „high grade" Tumor Budding signifikant reduziert ist (Gesamtüberleben nach 5 Jahren $-25 \%$ (95\%-Konfidenzintervall (-KI) -18 bis $-33 \%$; $p<0,00001)$; [20]). Der prädiktive Wert von Tumor Budding für eine adjuvante Chemotherapie ist jedoch bisher nicht sicher belegt. In aktuellen Studien konnte lediglich ein bisher nicht-signifikanter Trend für ein besseres Ansprechen von Karzinomen mit hohem Tumor Budding auf adjuvante Therapie beschrieben werden [24]. Es wurde jedoch gezeigt, dass das Rezidivrisiko nach Chemotherapie bei Patienten mit "high grade" Tumor Budding reduziert ist. Aktuell besteht keine grundsätzliche Indikation zur adjuvanten Chemotherapie bei Stadium-II-Karzinomen aufgrund des geringen Nutzens gegenüber den Nebenwirkungen (QUASARStudie), und diese wird nur bei einzelnen Subgruppen, wie etwa bei hoher Gradierung, pT4 oder extramuraler Veneninvasion in Erwägung gezogen [19]. Aufgrund des negativen prognostischen Werts von hohem Tumor Budding könnte es eine weitere Stütze für die klinische Entscheidung für eine adjuvante Therapie sein, jedoch ist die aktuelle Evidenz hierfür noch nicht ausreichend.

\section{Anwendungsbereich in präoperativen Biopsien}

Neben dem etablierten peritumoralen Budding in der Infiltrationszone eines Karzinoms wurde in mehreren aktuellen Studien "intratumorales Tumor Budding"
(ITB) in Biopsien mit den pathologischen Befunden der konsekutiven Resektionspräpaten korreliert [12, 16, 29]. Beispielsweise wurde beschrieben, dass hohes Tumor Budding in Biopsien signifikant korreliert ist mit einem höheren $\mathrm{N}$-Stadium $(p=0,034)$, Fernmetastasen $(p=0,007)$, Haemangiosis carcinomatosa $(p=0,046)$, Lymphgefäßinvasionen $(p=0,019)$ und infiltrativem Wachstum $(p<0,001 ;[12])$. Vor einer chirurgischen Resektion könnte aufgrund dieser Korrelation ein hohes Tumor Budding mit einer insgesamt schlechteren Prognose assoziiert sein und deshalb als Stütze für die klinische Entscheidung einer neoadjuvanten Therapie verwendet werden. Speziell beim Rektumkarzinom könnte daher eine neoadjuvante Therapie bei hohem Tumor Budding zu empfehlen sein. Bislang wurde in einer Studie niedriges Tumor Budding in präoperativen Rektumbiopsien mit einer kompletten pathologischen Tumorregression am Resektat assoziiert [16]. Hierfür sind jedoch weitere Studien nötig, um die Bedeutung von ITB zu verifizieren und Mindestanforderungen an das Biopsiematerial zu formulieren (Anzahl und Größe der Fragmente usw.).

\section{Ungeklärte und/oder schwierige Situationen bei der Tumor- Budding-Bestimmung}

1. „Pseudobuds": Eine Schwierigkeit für die Bestimmung der Zahl von Tumor Buds können sog. „pseudobuds" sein. Diese entstehen durch peritumorale Inflammation, welche zur Destruktion von Drüsengewebe des CRC führt. Aus diesem Grund sollte Tumor Budding nur in Areale ausgewertet werden, in welchen keine ausgeprägte Inflammation mit Destruktion von Tumordrüsen auftritt.

2. Muzinöses und siegelringzelliges Karzinom: Tumoreinzelzellen und Siegelringzellen, welche freischwebend in Muzin liegen und auch einzeln auftreten können, sollten nicht als Tumor Buds gewertet werden. Tumor Buds sollten als solche gezählt werden, wo sie von Stroma umgeben sind. In Abwesenheit jeglicher solcher Areale sollte mit einem entsprechenden Kommen- 

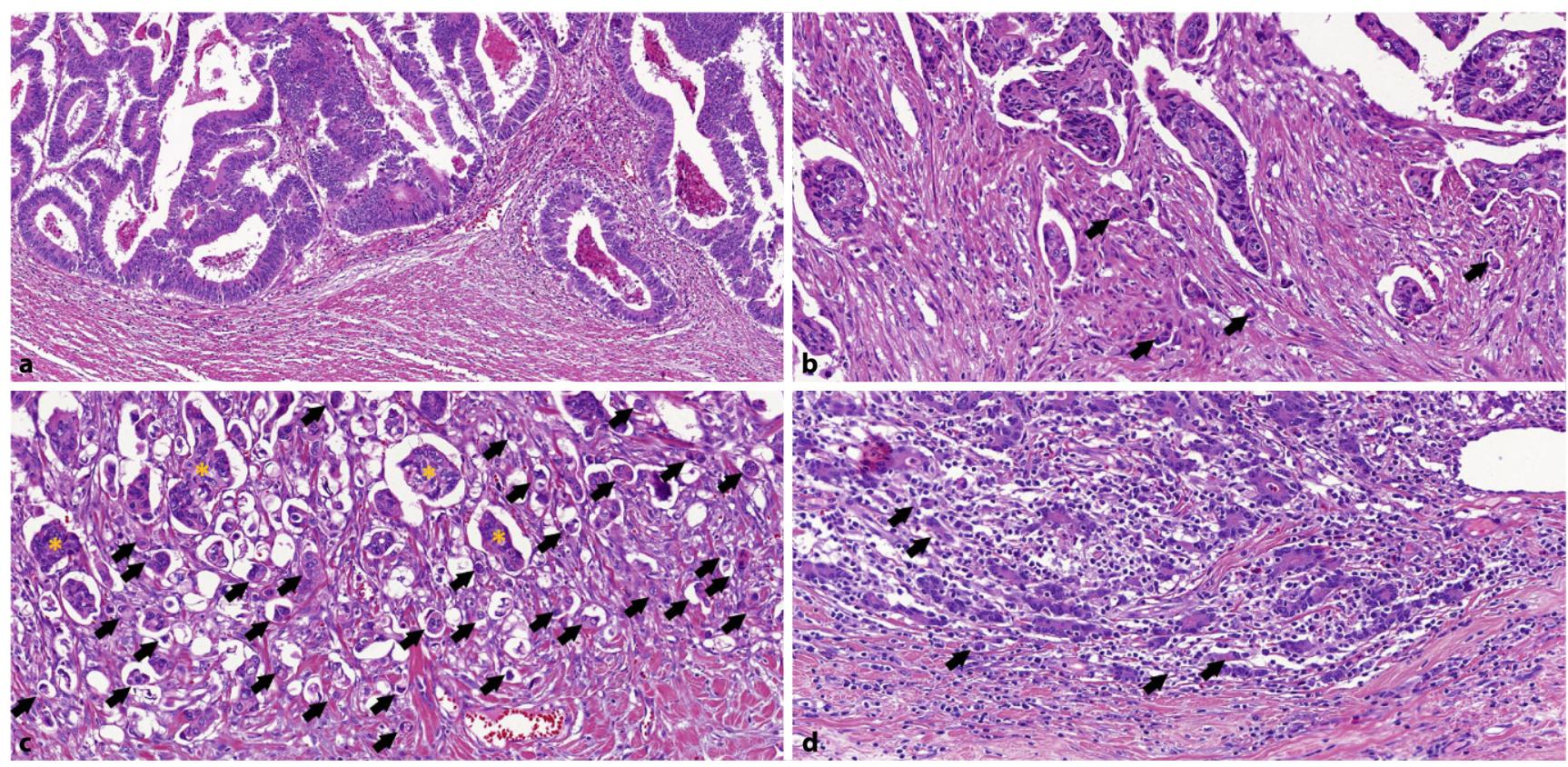

Abb. $1 \Delta$ Exemplarische Darstellung der drei Grade von Tumor Budding an Hämatoxylin/Eosin-Schnitten (ausgewählte TumorBuds mit Pfeilen gekennzeichnet): a Bd1 (0-4Buds $/ 0,785 \mathrm{~mm}^{2}$ ). b Bd2 (5-9Buds $/ 0,785 \mathrm{~mm}^{2}$ ). cBd3 (> 10Buds/0,785 mm²). In diesem Fall finden sich ebenfalls zahlreiche PDC („poorly differentiated clusters“, Sterne) und ein mikropapilläres Wachstumsmuster, welche für die Auswertung von Tumor Buds abzugrenzen sind. $\mathbf{d}$ Ein prominentes Entzündungsinfiltrat kann die Erkennung von Tumor Buds erschweren. Hierzu kann eine Panzytokeratinfärbung durchgeführt werden, um Tumor Buds zu identifizieren, die Auswertung sollte jedoch am HE erfolgen

tar kein Tumor Budding angegeben werden.

3. Überlagerung durch Entzündungsinfiltrate (• Abb. 1): Bei dichten peri-/ intratumoralen Entzündungsinfiltraten kann die Erkennung von Tumor Buds deutlich erschwert sein. In diesen Fällen ist es zulässig, zu Orientierungszwecken eine Zytokeratinfärbung durchzuführen, die eigentliche Auszählung sollte aber an der Hämatoxylin/ Eosin-Färbung stattfinden [14, 17].

4. Zustand nach neoadjuvanter Therapie: Bislang gibt es keine ausreichende Studienlage, ob Tumor Budding nach neoadjuvanter Therapie eine prognostische Aussage hat. Entsprechend der aktuellen Datenlage sollte Tumor Budding in diesen Fällen noch nicht berichtet werden [14].

5. "Poorly differentiated clusters" (PDC) und mikropapilläres Karzinom: Tumor Buds sind grundsätzlich von PDC abzugrenzen, welche definitionsgemäß aus $\geq 5$ Zellen bestehen und kein glanduläres Wachstumsmuster zeigen. Auch wenn Tumor Budding separat rapportiert werden soll, ist von einem biologischen Kontinuum zwischen
Tumor Buds, PDC und der mikropapillären Variante des CRC auszugehen (• Abb. 1; [6]). Diese Aspekte werden allerdings noch nicht in den bestehenden Scoringsystemen widergespiegelt. Die Verwendung des Cut-off von fünf Tumorzellen zwischen Tumor Buds und PDC basiert auf der großen Anzahl veröffentlichter Studien mit dieser Definition [17]. Es besteht jedoch wenig Evidenz dafür, inwieweit dieser Cutoff geeignet ist, beide Entitäten sicher voneinander zu differenzieren.

6. Interobserver-Variabilität: Die genannten schwierigen Situationen und die Tatsache, dass Tumor Buds ausgezählt (und nicht als vorhanden bzw. nicht detektiert angegeben) werden, können dazu führen, dass zwischen verschiedenen Untersuchern unterschiedliche Werte für das Tumor Budding bestimmt werden. Die Interobserver-Variabilität lässt sich durch Ausbildung und Erfahrung reduzieren, ist aber zugleich ein potenzielles Feld für die Anwendung von automatisierten Algorithmen in der digitalen Pathologie [23].

\section{Ausblick nach der ITBCC 2016}

Bereits bei der ITBCC 2016 war klar, dass der verfasste Konsens bei Erweiterung der Datenlage und adäquater Evidenz in der Literatur revidiert und verfeinert werden sollte. Beispielsweise wurde kürzlich eine zusätzliche Kategorie für vollständige Abwesenheit von Tumor Buds (Bd0; [26]) vorgeschlagen. In der publizierten Studie konnte gezeigt werden, dass CRC ohne Tumor Buds tendenziell niedrigere T-Stadien und eine bessere Prognose zeigten, so dass möglicherweise die Bd0-Kategorie zusätzlich eingeführt werden könnte, um Karzinome mit günstigerem Verlauf besser charakterisieren zu können.

Ebenso sollte die mögliche automatisierte Auswertung von Tumor Budding mittels digitaler Algorithmen in künftige Richtlinien mit einbezogen werden [14, 23].

\section{Fazit für die Praxis}

- Im Zuge der Standardisierung durch die International Tumor Budding Consensus Conference (ITBCC) 2016 ist Tumor Budding ein in der Praxis einfach bestimmbarer und morphologischer Score, welcher 
als zusätzlicher Risikofaktor neben der TNM-Klassifikation eine Aussagekraft hinsichtlich der Prognose eines kolorektalen Karzinoms (CRC) hat. Im geeigneten klinischen Kontext (endoskopische Resektion von pT1-Karzinomen und Stadium-II-CRC) kann Tumor Budding ein Hilfsmittel für die Therapieentscheidung sein. Tumor Budding könnte zukünftig in präoperativen Biopsien ebenfalls eingesetzt werden, dies muss allerdings weiter untersucht werden.

- Für die korrekte Bestimmung des Tumor Buddings ist es notwendig, die bestehende Konsensusmethode und oben beschriebenen potenzielle Schwierigkeiten in der Bestimmung zu kennen. Weitere Studien sollten diese Aspekte und offene Fragen gezielt erforschen um in überarbeitete Richtlinien integriert zu werden.

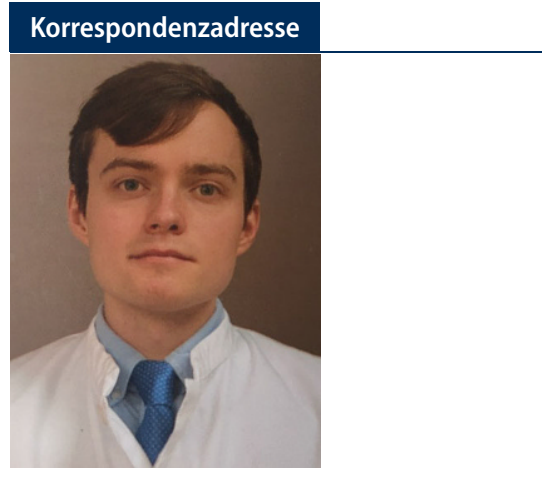

\section{Dr. med. Felix Müller}

Institut für Pathologie, Universität Bern Murtenstraße 31, 3008 Bern, Schweiz felix.mueller@pathology.unibe.ch

Danksagung. Die Autoren möchten sich bei Dr. med. Corinna Lang-Schwarz und Prof. Dr. med. Michael Vieth, Institut für Pathologie, Klinikum Bayreuth bedanken für die Nutzung histologischer Schnittpräparate zur Erstellung des Bildmaterials.

Funding. Open access funding provided by University of Bern

\section{Einhaltung ethischer Richtlinien}

Interessenkonflikt. F. Müller, A. Lugli und H. Dawson geben an, dass kein Interessenkonflikt besteht.

Für diesen Beitrag wurden von den Autoren keine Studien an Menschen oder Tieren durchgeführt. Für die aufgeführten Studien gelten die jeweils dort angegebenen ethischen Richtlinien.

Open Access. Dieser Artikel wird unter der Creative Commons Namensnennung 4.0 International Lizenz veröffentlicht, welche die Nutzung, Vervielfältigung, Bearbeitung, Verbreitung und Wiedergabe in jeglichem Medium und Format erlaubt, sofern Sie den/die

\section{Tumor budding in colorectal cancer-Information for clinical use and instructions for practical evaluation}

Background: Some patients with high-risk colorectal cancer show a worse prognosis within the same UICC stage. Therefore, the identification of additional risk factors is necessary to find the best treatment for these patients.

Objective: In which settings can tumor budding help the clinical decision-making process for treatment planning and how should scoring be performed?

Material and methods: Evaluation of current publications on tumor budding with an emphasis on practical grading and potential problems in the determination of tumor budding.

Results: Tumor budding is a significant risk factor for worse clinical outcome of colorectal cancer and can influence clinical decision-making in pT1 and stage II colorectal cancer. A scoring method was standardized by the ITBCC 2016 and is feasible in everyday practice. Challenges in assessment can be addressed by increasing awareness of potential problem cases.

\section{Keywords}

Lymph node metastasis · Epithelial-mesenchymal transition · Tumor cell clusters · Treatment planning · Tumor grading

ursprünglichen Autor(en) und die Quelle ordnungsgemäßnennen, einen Link zur Creative Commons Lizenz beifügen und angeben, ob Änderungen vorgenommen wurden.

Die in diesem Artikel enthaltenen Bilder und sonstiges Drittmaterial unterliegen ebenfalls der genannten Creative Commons Lizenz, sofern sich aus der Abbildungslegende nichts anderes ergibt. Sofern das betreffende Material nicht unter der genannten Creative Commons Lizenz steht und die betreffende Handlung nicht nach gesetzlichen Vorschriften erlaubt ist, ist für die oben aufgeführten Weiterverwendungen des Materials die Einwilligung des jeweiligen Rechteinhabers einzuholen.

Weitere Details zur Lizenz entnehmen Sie bitte der Lizenzinformation auf http://creativecommons.org/ licenses/by/4.0/deed.de.

\section{Literatur}

1. Ahn JH, KwakMS, Lee HH etal (2021) Development of a novel prognostic model for predicting lymph node metastasis in early colorectal cancer: analysis based on the surveillance, epidemiology, and end results database. Front Oncol 11:614398

2. Al. BaE (2021) NCCN clinical practice guidelines in oncology-colon cancer version 2.2021. https:// www.nccn.org/professionals/physician_gls/pdf/ colon.pdf.Zugegriffen: 10. Aug. 2021

3. Schmiegel WP S3-Leitlinie Kolorektales Karzinom. https://www.leitlinienprogramm-onkologie.de/ fileadmin/user_upload/Downloads/Leitlinien/ Kolorektales_Karzinom/Version_2/LL_KRK_ Langversion_2.1.pdf.Zugegriffen:11.Aug. 2021

4. Anonymous (2017) TNM Classfication of Malignant Tumors.

5. Argiles G, Tabernero J, Labianca R et al (2020) Localised colon cancer: ESMO Clinical Practice Guidelines for diagnosis, treatment and follow-up. Ann Oncol 31:1291-1305

6. Barresi V, Branca G, Vitarelli E et al (2014) Micropapillary pattern and poorly differentiated clusters represent the same biological phenomenon in colorectal cancer: a proposal for a change in terminology. Am JClin Pathol 142:375-383

7. Beaton C, Twine CP, Williams GL et al (2013) Systematic review and meta-analysis of histopathological factors influencing the risk of lymph node metastasis in early colorectal cancer. Colorectal Dis 15:788-797

8. Bosch SL, Teerenstra S, De Wilt JH et al (2013) Predicting lymph node metastasis in pT1 colorectal cancer: a systematic review of risk factors providing rationale for therapy decisions. Endoscopy 45:827-834

9. Brenner H, Kloor M, Pox CP (2014) Colorectal cancer. Lancet 383:1490-1502

10. Burgart LC, Chopp WV, Jain D (2021) Protocol for the examination of resection specimens from patients with primary carcinoma of the colon and rectum. https://documents.cap.org/protocols/ ColoRectal_4.2.0.0.REL_CAPCP.pdf. Zugegriffen: 11. Aug. 2021

11. Cappellesso R, Luchini C, Veronese $N$ et al (2017) Tumor budding as a risk factor for nodal metastasis in pT1 colorectal cancers: a meta-analysis. Hum Pathol 65:62-70

12. Giger OT, Comtesse SC, Lugli A et al (2012) Intra-tumoral budding in preoperative biopsy specimens predicts lymph node and distant metastasis in patients with colorectal cancer. Mod Pathol 25:1048-1053

13. Grigore AD, Jolly MK, Jia D et al (2016) Tumor budding: the name is EMT. Partial EMT. J Clin Med 5(5):51

14. Haddad TS, Lugli A, Aherne $S$ et al (2021) Improving tumor budding reporting in colorectal cancer: a Delphi consensus study. Virchows Arch 479(3):459-469

15. Imai T (1949) Histological comparison of cancer of the stomach in autopsy and operation cases. Jpn J Cancer Res 40:199-201

16. Lino-Silva LS, Gamboa-Dominguez A, ZunigaTamayo D et al (2018) Mismatch repair protein expression and intratumoral budding in rectal cancer are associated with an increased pathological complete response to preoperative chemoradio- 


\section{Schwerpunkt: Gastrointestinal Pathologie}

therapy: A case-control study. World J Clin Oncol 9:133-139

17. Lugli A, Kirsch R, Ajioka Y et al (2017) Recommendations for reporting tumor budding in colorectal cancer based on the International Tumor Budding Consensus Conference (ITBCC) 2016. Mod Pathol 30:1299-1311

18. Lugli A, Zlobec I, Berger MD et al (2021) Tumour budding in solid cancers. Nat Rev Clin Oncol 18:101-115

19. Mitrovic B, Handley K, Assarzadegan N et al (2021) Prognostic and predictive value of tumor budding in colorectal cancer. Clin Colorectal Cancer 20(3):256-264

20. Petrelli F, Pezzica E, Cabiddu M et al (2015) Tumour budding and survival in stage II colorectal cancer: a systematic review and pooled analysis. J Gastrointest Cancer 46:212-218

21. Rau TT, Bettschen E, Buchi Cet al (2021) Prognostic impact of tumor budding in endometrial carcinoma within distinct molecular subgroups. Mod Pathol 34:222-232

22. Rogers AC, Winter DC, Heeney A et al (2016) Systematic review and meta-analysis of the impact of tumour budding in colorectal cancer. Br J Cancer 115:831-840

23. Studer L, Blank A, Bokhorst JM et al (2021) Taking tumour budding to the next frontier-a post International Tumour Budding Consensus Conference (ITBCC) 2016 review. Histopathology 78:476-484

24. Ueno $H$, Ishiguro $M$, Nakatani $E$ et al (2019) Prospective multicenter study on the prognostic and predictive impact of tumor budding in stage II colon cancer: results from the SACURA trial. J Clin Oncol 37:1886-1894

25. Ueno H, Mochizuki H, Hashiguchi Y et al (2004) Risk factors for an adverse outcome in early invasive colorectal carcinoma. Gastroenterology 127:385-394

26. Zlobec I, Bachli M, Galuppini F et al (2021) Refining the ITBCC tumor budding scoring system with a "zero-budding" category in colorectal cancer. Virchows Arch. https://doi.org/10.1007/s00428021-03090-w

27. Zlobecl,BergerMD, Lugli A (2020) Tumourbudding and its clinical implications in gastrointestinal cancers. Br J Cancer 123:700-708

28. Zlobec I, Dawson HE, Blank A et al (2020) Are tumour grade and tumour budding equivalent in colorectal cancer? A retrospective analysis of 771 patients. Eur J Cancer 130:139-145

29. Zlobec I, Hadrich M, Dawson H et al (2014) Intratumoural budding (ITB) in preoperative biopsies predicts the presence of lymph node and distant metastases in colon and rectal cancer patients. Br JCancer 110:1008-1013

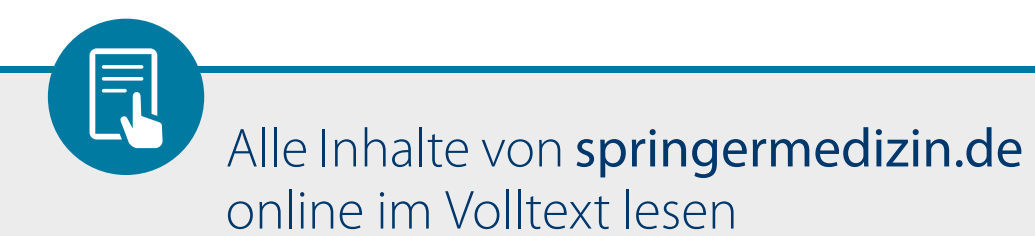

Mit e.Med Interdisziplinär - dem Kombi-Abo von SpringerMedizin.de können Sie jederzeit auf alle Inhalte zugreifen, die Sie für Ihren Praxis- oder Klinikalltag benötigen. Sie sind immer aktuell informiert - über die neuesten Publikationen, wichtige Studien oder innovative Therapieverfahren.

Die Vorteile von e.Med Interdisziplinär:

- Gestalten Sie Ihre Fortbildung nach Ihren Bedürfnissen: e.Med Interdisziplinär bietet Ihnen Online-Zugang zu 600 CME-Fortbildungskursen aller Fachrichtungen

- Sie entscheiden, was Sie lesen möchten:

Alle Fachzeitschriften stehen digital im Volltext zur Verfügung.

- Finden Sie die gewünschten Informationen auch in englischen Publikationen: Sie können in mehr als 500 englischsprachigen Fachzeitschriften online recherchieren und auf die Suchergebnisse uneingeschränkt im Volltext zugreifen.

- Auf Wunsch erhalten sie darüber hinaus eine gedruckte Fachzeitschrift nach Wahl.

Mit den fachspezifischen e.Med-Abos können Sie sich effizient innerhalb Ihres Fachgebietes auf dem Laufenden halten. Das Angebot reicht von AINS bis Radiologie - Sie haben die Wahl!

Testen Sie e.Med kostenlos und unverbindlich!

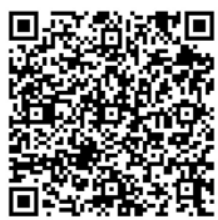
Jetzt informieren unter www.springermedizin.de $\Rightarrow$ "Abos" oder telefonisch unter 0800-77 80777

(Montag bis Freitag, 10 bis 17 Uhr) 